\title{
Evaluating the Effects of Antimicrobial Drug Use On the Ecology of Antimicrobial Resistance and Microbial Community Structure in Beef Feedlot Cattle
}

\section{Enrique Doster}

Colorado State University https://orcid.org/0000-0002-3820-8988

Lee J. Pinnell

Texas A\&M University

Noelle R. Noyes

University of Minnesota College of Veterinary Medicine Jennifer K. Parker

The University of Texas at Austin

Cameron A. Anderson

Colorado State University

Calvin W. Booker

Feedlot health management services

Sherry J. Hannon

Feedlot Health Management Services

Tim A. McAllister

Agriculture and Agri-Food Canada

Sheryl P. Gow

Public Health Agency of Canada

Keith E. Belk

Colorado State University

Paul S. Morley ( $\square$ pmorley@cvm.tamu.edu )

Texas A\&M University https://orcid.org/0000-0001-8138-2714

\section{Research}

Keywords: Resistome, Microbiome, Microbial ecology, Antimicrobial drug exposure, Antimicrobial resistance

Posted Date: November 1st, 2021 
DOI: https://doi.org/10.21203/rs.3.rs-1022179/v1

License: (c) (1) This work is licensed under a Creative Commons Attribution 4.0 International License. Read Full License 


\section{Abstract}

\section{Background}

Antimicrobial drugs (AMDs) are used in beef production to treat clinical disease and to prevent or control infections in groups of cattle. Use of AMDs in food producing animals has received increasing scrutiny because of concerns about antimicrobial resistance (AMR) that might affect consumers. Previously, investigations regarding AMR have focused largely on phenotypes of selected pathogens and indicator bacteria, but genes that confer AMR are known to be distributed and shared throughout microbial communities. Use of high-throughput metagenomic sequencing provides a holistic perspective on AMR ecology by examining determinants within the entire microbiome. The primary objective of this study was to employ metagenomic sequencing to investigate the effects of AMD use on the microbiome and resistome in beef feedlot cattle.

\section{Results}

This study leveraged the use of archived samples that were collected during a previous longitudinal study of cattle at beef feedlots in Canada. This included fecal samples collected from randomly selected individual cattle, as well as composite-fecal samples from randomly selected pens of cattle. All AMD use was recorded and characterized across different drug classes using animal defined daily dose (ADD) metrics. Samples were analyzed using AMR target-enriched shotgun sequencing to characterize the fecal resistome and 16S rRNA gene sequencing to characterize the microbiome. Overall, the fecal resistome composition was dominated by alignments to gene accessions conferring resistance to tetracycline and macrolide-lincosamide-streptogramin (MLS) drug classes. The diversity of bacterial phyla was greater early in the feeding period and decreased over time as the microbiome shifted toward a similar composition dominated by Proteobacteria and Firmicutes. Antimicrobial drug exposures in individuals and groups were associated with explaining a statistically significant proportion of the variance in the resistome, but their contribution to the variance was small compared to other factors measured in this study.

\section{Conclusions}

Time in the feedlot was associated with greater changes in the microbiome and resistome for both individual animals and pen-floor samples, although the proportion of the variance associated with this factor was small. Results of this study are consistent with other investigations showing that AMD exposures did not have strong effects on the microbial ecology of beef cattle.

\section{Background}

Antimicrobial resistance (AMR) is one of the most critical global public health issues (World Health Organization [WHO], 2015, 2017, 2019; Centers for Disease Control and Prevention [CDC], 2019, 2020). Misuse and overuse of antimicrobial drugs (AMDs) are commonly attributed as the principal drivers of 
this problem, and many believe that these practices are accelerating the development and spread of AMR (WHO, 2015, 2019; CDC, 2019). The use of AMDs in food-producing animals has received increasing criticism and scrutiny regarding this critical issue. Some propose that the use of AMDs within agriculture poses an unacceptable risk to public health because of the potential promotion of AMR, and the potential distribution of resistant bacteria either directly via food products, or indirectly through contamination of soil, water, and air (WHO, 2015, 2019; CDC, 2019, 2020).

Traditionally, research and surveillance for AMR have relied on aerobic culture to isolate bacterial isolates which then undergo in vitro phenotypic assessments of resistance to various drugs (i.e., using broth microdilution and disk diffusion). While informative, results from these types of studies only provide information about AMR patterns for the very limited number of culturable microorganisms, and for a few AMDs. These culture-based approaches have a limited ability to provide a holistic perspective on resistance in entire microbial communities (microbiomes). Evaluating AMR within an ecological, community-based context is critical, as resistance genes are not limited to a single species or strain but are found within many microbial taxa in the microbiome (e.g., Brinkac et al., 2017; Ghosh et al., 2013). Further, resistance genes can be rapidly transferred between microorganisms via horizontal gene transfer or mobile genetic elements (von Wintersdorff et al., 2016; Zhang et al., 2021). Thus, the entire reservoir of resistance genes (the resistome) can be considered to have a related but potentially separate ecology from the microbiome and should be investigated within the context of whole microbial communities.

The composition of the microbiome and resistome can be investigated in a comprehensive manner without the need to culture specific bacteria through next-generation sequencing (NGS). The most common statistical approaches to analyze NGS data use summary statistics and dimension-reduction techniques such as diversity indices that are common in ecological studies (e.g., principal coordinate analyses). The primary goal of this study was to characterize the impact of AMD exposures on the fecal microbiome and resistome of beef feedlot cattle. This study used AMR target-enriched metagenomic sequencing and 16S rRNA gene sequencing to investigate the effects of AMD use on the fecal resistome and microbiome of cattle at four Canadian feedlots with detailed AMD use records (Benedict et al., 2013; Noyes et al., 2016a).

\section{Methods}

\section{Study design and sample collection}

Feces were collected during a 3-year longitudinal study of Canadian beef feedlot operations, and results regarding in vitro susceptibility of Escherichia coli and Mannheimia haemolytica cultured from these fecal samples have been previously described (Benedict et al., 2015; Noyes et al., 2015, 2016a). Briefly, cattle were enrolled from September 2007 to January 2010 using two-stage random sampling. As cattle arrived at the feedlots, $30 \%$ of all newly formed pens were randomly selected for inclusion, and approximately $10 \%$ of all cattle housed in selected pens were randomly enrolled. 
Individual cattle were sampled twice over the course of the study: during initial processing, which occurred shortly after arrival to the feedlot (first sample), and later in the feeding period (second sample) when cattle were rehandled for various standard management protocols (e.g., for replacement of growthpromoting implants). The timing for obtaining the second samples from cattle varied from 33-202 dayson-feed (DOF) with an average of 95.5 DOF (median = 80.0 DOF). Composite fecal samples were collected from floors of pens soon after occupancy (first sample), near the dates when individual animal samples were collected from animals assigned to that pen (second sample time point), and just prior to slaughter (third sample time point). Composite samples were comprised of approximately $1 \mathrm{gram}$ of feces collected from 20 fresh fecal pats, which were placed in a sterile container and then mixed thoroughly. After collection, feces were placed in Cary-Blair media and refrigerated for transport to the laboratory (Benedict et al., 2013). At the laboratory, fecal samples were processed for aerobic culture and in vitro susceptibility testing as previously described, and then archived at $-80^{\circ} \mathrm{C}$. These samples were archived as described for approximately 10 years prior to being processed for this study. All exposures of the study population to antimicrobial drugs, including parenteral treatments and in-feed exposures, were recorded and standardized across different drug classes using animal defined daily dose estimates (ADD) (Benedict et al., 2015; Noyes et al., 2015).

\section{Sample selection for metagenomic sequencing}

For this investigation of the fecal microbiome and resistome, a subset of all individual animal and composited pen-floor fecal samples collected for the previous study were randomly selected for use in this metagenomic sequencing study, after stratifying individual cattle based upon cumulative AMD exposures prior to sampling, and stratifying pen-floor samples on the timing of sample collection. Samples from both the first and second sampling time point had to be available for testing in order for individual cattle to be eligible for inclusion. Twenty eligible cattle were selected from each of 3 parenteral ADD exposure categories: cattle with no AMD exposure prior to the second time point, those exposed parenterally to 1-4 ADDs prior to the second time point, and those exposed to $>4$ ADDs parenterally. Thus, the total subset included 60 samples collected from individual cattle sampled at each of the first and second time points. However, of the 120 individual-animal fecal samples selected, only 94 were successfully sequenced using AMR targeted-enriched sequencing to characterize the resistome because insufficient amounts of DNA were available after DNA extraction to meet the minimum requirements for library preparation (total DNA yield greater than $9 \mu \mathrm{g}$ ).

For investigation of pen-level samples, archived composite samples had to be available from both the first and second time point in order for a pen to be eligible for inclusion in this study. Because the total ADDs for AMD exposure were relatively low among eligible pens, the 6 pens that had the highest total accumulated ADDs were purposively selected for inclusion. The remaining eligible pens were stratified on whether the second sample was collected before or after $100 \mathrm{DOF}$, and 19 pens were randomly selected from each of these strata for inclusion. As previously described (Noyes et al., 2016a), a limited number of pens were sampled at the third time point, and 10 of these samples were randomly selected for inclusion. 
Thus, the total subset included 44 samples collected at each of the first and second time points, and 10 samples collected at the third time point.

\section{DNA extraction}

Genomic DNA was isolated from $5 \mathrm{~g}$ of feces using the Qiagen PowerMax Soil Kit (Qiagen Laboratories) according to manufacturer's instructions, with two exceptions to increase yield. First, samples were centrifuged for 5 minutes in the PowerMax bead tubes, as opposed to the recommended 3 minutes. Second, samples were eluted using $3 \mathrm{~mL}$ as opposed to the recommended $5 \mathrm{~mL}$ and were passed through the silica DNA filter twice. To increase DNA concentration, isolated DNA was precipitated with ethanol and $0.3 \mathrm{M}$ sodium acetate, washed with $70 \%$ ethanol, and resuspended in $150 \mu \mathrm{L}$ of PowerMax elution buffer. DNA was then quantified in duplicate using the Qubit 2.0 Fluorometer and dsDNA High Sensitivity Buffer and Reagent kit (Thermo Fischer Scientific) and a final concentration was calculated by averaging the two measurements. DNA was also assayed for quality $\left(A_{260} / A_{280}\right.$ and $\left.A_{260} / A_{230}\right)$ using a NanoDrop 1000 Spectrophotometer (Thermo Fischer Scientific). If a sample failed to reach a total DNA yield greater than $9 \mu \mathrm{g}$, it was extracted a second time and combined with the first. If there was not $5 \mathrm{~g}$ of sample remaining for the second extraction, sterile PBS was used to recover remaining feces off the transport tubes that samples were stored in. The volume of PBS used was dependent upon the weight of the remaining sample; more PBS was used for samples with less weight, in order to reach a total of $5 \mathrm{~g}(\mathrm{w} / \mathrm{v})$ extraction volume.

\section{Library preparation and sequencing}

The SureSelectXT HS Reagent Kit for Illumina Paired-End Multiplexed Sequencing Library (Agilent Technologies) was used to prepare samples for target-enriched resistome sequencing. A custom bait design targeting AMR genes, 'MEGaRICH' (Noyes et al., 2017) was used to enrich sequencing libraries for AMR gene sequences. Resulting libraries were pooled in equal proportions based on their molecular weight and DNA concentrations. The pooled library was sequenced at the Denver Genomics and Microarray Core Facility (Denver, CO) on an Illumina NovaSeq 6000 instrument using paired-end chemistry $(2 \times 150 \mathrm{bp})$ and a targeted read depth of 15 million reads per sample.

Extracted DNA (200 - 500ng) from each sample $(n=218)$ was sent to the Novogene Corporation for $16 S$ rRNA gene amplification, library preparation, and sequencing. The V4 region of the 16S rRNA gene was amplified using the 515F/806R primer pair [5'-GTGCCAGCMGCCGCGGTAA-3']/[5'-

GGACTACHVGGGTWTCTAAT-3']. Amplicon sequencing was performed on an Illumina HiSeq 2500 instrument using paired-end chemistry $(2 \times 250 \mathrm{bp})$ and a targeted read depth of 100,000 reads per sample.

\section{Resistome and microbiome characterization}

Target-enriched AMR metagenomic sequencing reads were processed using the AMR++ v2 bioinformatic pipeline and the MEGARes v2 resistance database (Doster et al., 2020)(Lakin et al., 2017). A detailed description of MEGARes and the AMR++ pipeline can be found at http://megares.meglab.org. Briefly, 
reads were trimmed and filtered for quality using trimmomatic (Bolger et al., 2014), and bovine host DNA was identified by aligning trimmed reads to the Bos Taurus genome with BWA (Li \& Durbin 2009) and removing those aligned reads. Using BWA, reads were aligned to the MEGARes database and with samtools, alignments were then de-duplicated to remove reads with $100 \%$ similarity. Only genes with reads aligning to $>80 \%$ of the reference nucleotide sequence were considered for further analysis, with the exception of reads aligned to genes that require the presence of specific single nucleotide polymorphisms to confer resistance. These reads were identified, removed from the downstream statistical analysis, and described separately. Additionally, a list of important AMR gene determinants in human-associated pathogens were identified a priori and searched for in all samples. These included genetic determinants classified as Class A carbepenemases [bla(IMI), bla(SME), bla(GES), bla(KPC)], Class B carbapenemases [bla(NDM), bla(IMP), bla(cph)], Class D carbapenemases [bla(OXA)], extended spectrum betalactamase [bla(TEM), bla(SHV), bla(CTX-M), bla(CMY)], streptogramin resistance [vga/vgb/vat], colistin resistance [ $\mathrm{mcl}$, and multidrug resistance to phenicols, lincosamides, oxazolidinones, pleuromutilins, and streptogramin A antibiotics $\left(\mathrm{PhLOPS}_{\mathrm{A}}\right)[\mathrm{cff}]$.

16S rRNA amplicon sequence reads were analyzed using QIIME2 v2018.12 (Bolyen et al., 2019). Briefly, all reads were processed for sequence quality and denoised using DADA2 (Callahan et al., 2016), and the resulting amplicon sequence variants (ASVs) were classified using a Naïve Bayes classifier trained on the GreenGenes v13_8 database (DeSantis et al., 2006). Reads that mapped to chloroplast and mitochondrial DNA were then removed from the ASV count table.

\section{Processing of count matrices}

Following read processing with AMR++ or QIIME2, classification count matrices for AMR gene accessions and ASVs, respectively, were imported into R v3.5.1 (R Core Team, 2018). Cumulative sum scaling (CSS) (Paulson et al., 2013) was used to normalize feature counts and account for differences in sequencing depth using the R package 'metagenomeSeq' (Paulson et al., 2013). Sparsely represented features that were identified in fewer than $5 \%$ of samples were removed from further analysis based on published recommendations (Paulson et al., 2013). Resistance data was then summarized to the class and mechanisms level to avoid bias at the "gene" level associated with irregular naming criteria for resistance genes (Hall and Schwarz, 2016). Resistance determinants that affect multiple drug/compound classes (e.g., multi-compound resistance mechanisms such as multidrug efflux pumps) were categorized and analyzed as a separate resistance "class" (multidrug resistance - MDR). Because there is not an efficient method using high-throughput computational processing to confirm the presence of SNPs that are critical for conferring resistance in some specific genes, reads aligning to gene accessions that require SNP confirmation were excluded from statistical analysis (Supplemental File S4). For microbial community analyses, taxonomy was assigned at the level of phylum, class, order, family, genus, and species. However, to reduce the repetitive reporting of results at all levels and because results at lower taxonomic levels are not considered very reliable (Peabody et al., 2015), results are only presented at the phylum, class and order levels. Richness and Shannon's diversity indices were calculated for each sample using the "vegan" package. 


\section{Statistical analysis}

In alignment with the primary study goals, the exposures of interest for statistical analyses that were identified a priori were the total ADD exposures for AMDs, and the time that animals were in the feedlot prior to sampling (e.g., arrival vs second- or third-sampling timepoints). Because the second and third sampling timepoint occurred at a range of DOF timepoints, a separate variable categorizing the DOF at the time of sample collection was also classified into 5 categorical ranges (arrival to 3 DOF, 4-70 DOF, 71120 DOF, 121-180 DOF, and > 180 DOF). The total ADDs of AMD exposures for each animal and pen sample were calculated as the sum of ADDs from all sources prior to sample collection. However, the data regarding exposures to enrolled individuals versus entire pen groups were categorized separately. Total AMD exposures prior to sampling for individual animals were categorized into 3 ordinal levels based on ADDs: low exposure (<10), medium exposure (10 - 19, and high exposure $(>19)$. Total AMD exposures for pen groups were also categorized into 3 ordinal levels; low exposure $(<400)$, medium exposure $(400-1100)$, and high exposure $(>1100)$.

Diversity indices were statistically compared using the Wilcoxon signed-rank test ("wilcox.test" function in R) for samples from the same animal and "glm" to test differences between sample groups. CSSnormalized counts were Hellinger-transformed (Legendre and Gallagher, 2001) for ordination using the metaMDS function from "vegan", which employs non-metric multidimensional scaling on Euclidian distances. Analysis of similarities (ANOSIM) (Clarke, 1993) was used to test differences in the composition of microbial communities and resistomes between categorical metadata sample groups (e.g., ADD exposure category, arrival vs second- or third-sampling, and DOF sampling category). MetagenomeSeq's "fitZig" function was used to fit a zero-inflated Gaussian model and compare log2-fold differences (Paulson et al., 2013) in microbiome and resistome features between sampling time. Limma's "makeContrast" function (Ritchie et al., 2015) were then used for pairwise comparisons, P-values were adjusted for multiple tests using the Benjamini-Hochberg procedure (Benjamini and Hochberg, 1995), and alpha $=0.05$ was selected as the statistical significance cut-off value. To account for spurious statistically significant differences in low abundance features, only features with an average expression $>1 \%$ were considered.

Raw counts were Hellinger-transformed (Legendre and Gallagher, 2001) and redundancy analysis (RDA) was performed on microbial community and resistome composition to further evaluate the potential significance of different AMD use practices using the "rda" function in R. Significance of the correlation between independent variables and the variance in the microbiome and resistome composition were then tested using the "anova" function in R. To characterize the effect of ADD exposure and DOF on the microbiome and resistome, samples were grouped into 17 metadata categories for analysis. Values for ADD exposures were aggregated by route of administration (in-feed vs parenteral) and by drug class including macrolides-lincosamides-streptogramin (MLS), tetracyclines, phenicol, and bactrim (sulfamethoxazole and trimethoprim combination). Samples were summarized into metadata variables that reflect the amount and type of antimicrobial drug exposure as well as time in the feedlot and days since the most recent parenteral treatment (Supplemental File S1 \& S2). Variables regarding individual or 
pen status at the time of sampling included: feedlot ID (1-4), sampling time (first, second, third), DOF at the time of sampling, number of parenteral treatments with AMDs, total ADDs for exposures to all AMDs, total ADDs for exposures to tetracycline class drugs, total ADDs for tetracycline drugs administered in feed, ADDs for tetracycline drugs administered parenterally, total ADDs for exposures to MLS class drugs, ADDs for MLS drugs administered in feed, ADDs for MLS drugs administered parenterally, ADDs for parenteral exposure to phenicol class drugs, ADDs for parenteral exposures to phenicol class drugs, ADDs for parenteral exposures to sulfonamide class drugs. All variables were included in the starting model for step-wise backward variable selection and ANOVA testing was used to identify a best fitting model.

\section{Results}

\section{Individual animal sample sequencing metrics}

Across the 94 samples, AMR target-enriched metagenomic sequencing yielded an average of 15,926,612 paired-end reads (clusters) per sample (range 3.1M - 25.2M per sample, Supplemental File S1). Filtering to improve overall read quality and to exclude bovine host DNA removed an average of $22.9 \%$ of reads per sample (range: $3.0 \%$ - 38.5\%). Sequencing of the $16 \mathrm{~S}$ rRNA gene across 120 samples resulted in an average of 147,046 reads per sample (range 101,543 - 208,020 per sample, Supplemental File S1). Following quality filtering, identification of amplicon sequence variants (ASVs) with DADA2, and removal of chloroplast and chimeric sequences, samples averaged 40,855 ASVs (range 20,943-65,270 per sample, Supplemental File S1).

\section{Composite pen-floor sample sequencing metrics}

Across the 98 pen-floor composite samples, target-enriched AMR metagenomic sequencing resulted in an average of $16,470,078$ paired-end reads (clusters) per sample (range $6.2 \mathrm{M}-25.5 \mathrm{M}$ reads per sample, Supplemental File S2). Filtering to improve overall read quality and to exclude bovine host DNA removed an average of $18.7 \%$ of reads per sample (range: $3.3 \%-48.0 \%$, Supplemental File S1). $16 \mathrm{~S}$ rRNA gene sequencing resulted in an average of 197,889 paired -end reads (range 94,433 - 219,918 per sample, Supplemental File S2). After quality filtering, identification of ASVs with DADA2, samples averaged 50,426 ASVs (range 25,435 - 81,128 ASVs per sample).

\section{Resistome composition}

Across the 94 fecal samples collected from individual cattle, an average of 421,355 reads per sample were classified as genetic determinants of AMR (Supplemental File S3). The classifications represented 1,152 different published gene sequences that confer resistance to 18 different drug classes through 60 distinct resistance mechanisms. Across all sampling time points, the 9 most abundant drug classes (or multi-compound mechanisms) were tetracyclines (60.8\%), drug and biocide resistance (8.2\%), aminoglycosides (7.2\%), macrolide-lincosamide-streptrogramin (MLS - 5.6\%), betalactams $(5.5 \%)$, sulfonamides (4.2\%), phenicols (3.5\%), drug and biocide and metal resistance (2.6\%), and biocide and metal resistance (1.1\%). The remaining 9 classes each comprised less than $1 \%$ of all normalized counts. 
Genes conferring resistance to rifampin were identified in 13/94 samples and resistance to fluoroquinolones was identified in only $1 / 94$ fecal samples. Of the genes that confer tetracycline resistance, $89.1 \%$ represented tetracycline resistance ribosomal protection proteins, $8.4 \%$ were major facilitator superfamily (MFS) efflux pumps, and $2.5 \%$ were tetracycline inactivation enzymes. In the second most abundant group of resistance determinants, multi-compound drug and biocide resistance, $39 \%$ of alignments drug and biocide MFS efflux pumps and $30.8 \%$ represented drug and biocide RND efflux pumps.

In the 98 composite fecal samples collected from pen-floors, an average of 559,961 reads per sample were classified as genetic determinants of AMR (Supplemental File S3), representing 1,361 genes that confer resistance to 20 different drug classes through 69 distinct resistance mechanisms. Across all sampling time points, the 8 most abundant drug classes or MDR mechanisms were represented by tetracyclines (69.4\%), MLS (8.2\%), aminoglycosides (6.2\%), betalactams (4.6\%), multi-compound drug and biocide resistance (4\%), sulfonamides (2.8\%), phenicol (2.2\%), and drug and biocide and metal resistance mechanisms (1.1\%). The remaining 12 classes each consisted of $<1 \%$ of normalized counts (Figure 1). Gene conferring resistance to rifampin were identified in 15/98 samples, resistance to cationic antimicrobial peptides was identified in 5/98 samples, and fosfomycin, mupirocin, and fluoroquinolone resistance were only identified in a single sample each. Of the genes conferring tetracycline resistance, $89 \%$ encoded tetracycline resistance ribosomal protection proteins and $8.7 \%$ encoded for MFS efflux pumps with the remaining $2.4 \%$ associated with tetracycline inactivation enzymes. In the second most abundant resistance class, MLS, the two most abundant resistance mechanisms were 23S rRNA methyltransferases (49.7\%) and MLS resistance MFS efflux pumps (24.3\%).

\section{Changes in resistome composition over time}

There were significant shifts in richness and Shannon's diversity indices between sampling time points. At the class level, richness significantly decreased in individual animal samples $(W=1507.5, P=0.002)$ and in pen-floor composite samples $(\mathrm{W}=1412.5, \mathrm{P}<0.001)$. Shannon's diversity was also significantly different between sampling points in the individual animal samples $(W=1654, P<0.001)$ and in pen floor composite samples at the class level $(\mathrm{W}=1637, \mathrm{P}<0.001)$. Likewise, there was a statistically significant shift in resistome composition over time for fecal samples collected from both individual animals and pen-floor composite samples. The temporal shift in resistome composition was greater among samples collected from individual animals (class level: $A N O S I M R=0.33, P=0.001$; mechanism level: ANOSIM R = $0.34, P=0.001$; Figure $2 \mathrm{~A}$ ) than it was among pen-floor composite samples (class level: ANOSIM $R=$ $0.18, \mathrm{P}=0.001$; mechanism level: ANOSIM R $=0.18, \mathrm{P}=0.001$; Figure 2B). Temporal differences in the relative abundance of drug classes were more prominent among individual animal samples than penfloor composites, particularly due to decreases in the second most abundant resistance class, multicompound drug and biocide resistance. The pen-level resistome was dominated by tetracycline, and MLS resistance at the first sampling time point, and by the second sampling time point tetracycline resistance made up a greater proportion of the resistome in both sample types and significant shifts were limited to the drug classes in lower abundance (Figure 1). Of the 8 drug classes making up greater than $1 \%$ of the 
resistome, 8 were differentially abundant in individual animal samples while only 2 were differentially abundant in pen-floor composite samples (Supplemental File S5). Interestingly, shifts in the composition of individual animal resistomes were primarily the result of significant increases in the abundance of the three most prevalent drug classes (tetracyclines, MLS, and sulfonamides). In contrast, the abundance of these three drug classes did not change over time in pen-floor composite samples (Supplemental File S6). Instead, the 2 drug classes with significant changes in pen-floor composite samples were all associated with less prevalent drug classes that decreased in abundance. Notably classes consisting of multi-drug resistance, drug and biocide resistance, and drug and biocide and metal resistance mechanisms all decreased significantly over time in both individual animal samples and pen-floor composite samples.

\section{AMD exposures in the study population}

AMD exposures for the entire study population have previously been described (Benedict et al., 2015; Noyes et al., 2015). The average AMD exposures at the second timepoint in individual cattle selected for this study was 12.7 total ADDs (range $5.9-24.6$ ADD), which included an average parenteral exposure of 3.1 ADD (range 0 - 7 ADD - Table 1). Parenteral exposures were also dominated by tetracycline drugs (1.6 $A D D)$ and macrolide drugs (1.2 ADD), followed by phenicol (0.2 ADD), and sulfonamide drugs (0.1 ADD) on average. The majority of in-feed AMD exposures were to tetracyclines (average 9.6 ADDs per animal) compared to MLS (0.02 ADDs). Correspondingly, groups of cattle housed in enrolled pens were exposed to on average of 2,113.8 ADDs per pen (range $9.9-10,113.3$ ) at the second timepoint, consisting mostly of in-feed AMD exposures. In contrast, pens of cattle were exposed to average of 368.3 ADD by parenteral exposures (range $0-1367$ ADD) (Table 1). At the pen level, parenteral exposure to tetracycline drugs was most common, with an average of 140.9 ADD per pen (range $0-902)$. At one of the participating feedlots, parenteral exposure to fluoroquinolone drugs was more common in the enrolled pens $(n=6)$, accumulating an average of 1219.5 ADDs at the second time point. This is in comparison to an average of 57.8 fluoroquinolone ADDs in the other 49 pens. Without the influence of these 6 pens, tetracyclines made up the largest percentage of parenteral AMD exposures (65\%), followed by fluoroquinolone drugs (30\%), and betalactam drugs (2\%) with MLS, phenicol, and sulfonamide drugs each making up less than $1 \%$ of drug exposures. 
Table 1

- Summary statistics for ADD exposure variables, by sample type.

\begin{tabular}{|c|c|c|c|c|c|c|}
\hline $\begin{array}{l}\text { Sample } \\
\text { type }\end{array}$ & Variable & mean & median & $\min$ & $\max$ & sd \\
\hline $\begin{array}{l}\text { Individual - } \\
\text { 1st time } \\
\text { point }\end{array}$ & Total_ADD & 0.0 & 0.0 & 0.0 & 0.0 & 0.0 \\
\hline $\begin{array}{l}\text { Individual - } \\
\text { 1st time } \\
\text { point }\end{array}$ & Total_feed_ADD & 0.0 & 0.0 & 0.0 & 0.0 & 0.0 \\
\hline $\begin{array}{l}\text { Individual - } \\
1 \text { st time } \\
\text { point }\end{array}$ & Total_parenteral_ADD & 0.0 & 0.0 & 0.0 & 0.0 & 0.0 \\
\hline $\begin{array}{l}\text { Individual - } \\
1 \text { st time } \\
\text { point }\end{array}$ & total_tetracycline_ADD & 0.0 & 0.0 & 0.0 & 0.0 & 0.0 \\
\hline $\begin{array}{l}\text { Individual - } \\
\text { 1st time } \\
\text { point }\end{array}$ & total_MLS_ADD & 0.0 & 0.0 & 0.0 & 0.0 & 0.0 \\
\hline $\begin{array}{l}\text { Individual - } \\
\text { 1st time } \\
\text { point }\end{array}$ & feed_MLS_ADD & 0.0 & 0.0 & 0.0 & 0.0 & 0.0 \\
\hline $\begin{array}{l}\text { Individual - } \\
1 \text { st time } \\
\text { point }\end{array}$ & feed_tetracycline_ADD & 0.0 & 0.0 & 0.0 & 0.0 & 0.0 \\
\hline $\begin{array}{l}\text { Individual - } \\
1 \text { st time } \\
\text { point }\end{array}$ & parenteral_tetracycline_ADD & 0.0 & 0.0 & 0.0 & 0.0 & 0.0 \\
\hline $\begin{array}{l}\text { Individual - } \\
1 \text { st time } \\
\text { point }\end{array}$ & parenteral_MLS_ADD & 0.0 & 0.0 & 0.0 & 0.0 & 0.0 \\
\hline $\begin{array}{l}\text { Individual - } \\
\text { 1st time } \\
\text { point }\end{array}$ & parenteral_phenicol_ADD & 0.0 & 0.0 & 0.0 & 0.0 & 0.0 \\
\hline $\begin{array}{l}\text { Individual - } \\
\text { 1st time } \\
\text { point }\end{array}$ & parenteral_sulfonamide_ADD & 0.0 & 0.0 & 0.0 & 0.0 & 0.0 \\
\hline $\begin{array}{l}\text { Individual - } \\
1 \text { st time } \\
\text { point }\end{array}$ & parenteral_betalactams_ADD & 0.0 & 0.0 & 0.0 & 0.0 & 0.0 \\
\hline $\begin{array}{l}\text { Individual - } \\
\text { 1st time } \\
\text { point }\end{array}$ & parenteral_fluoroquinolones_ADD & 0.0 & 0.0 & 0.0 & 0.0 & 0.0 \\
\hline
\end{tabular}




\begin{tabular}{|c|c|c|c|c|c|c|}
\hline $\begin{array}{l}\text { Sample } \\
\text { type }\end{array}$ & Variable & mean & median & $\min$ & $\max$ & sd \\
\hline $\begin{array}{l}\text { Individual - } \\
\text { 2nd time } \\
\text { point }\end{array}$ & Total_ADD & 12.7 & 10.5 & 5.9 & 24.6 & 5.6 \\
\hline $\begin{array}{l}\text { Individual - } \\
\text { 2nd time } \\
\text { point }\end{array}$ & Total_feed_ADD & 9.6 & 7.0 & 3.9 & 18.7 & 5.4 \\
\hline $\begin{array}{l}\text { Individual - } \\
\text { 2nd time } \\
\text { point }\end{array}$ & Total_parenteral_ADD & 3.1 & 3.0 & 0.0 & 7.0 & 1.4 \\
\hline $\begin{array}{l}\text { Individual - } \\
\text { 2nd time } \\
\text { point }\end{array}$ & total_tetracycline_ADD & 11.2 & 9.9 & 5.9 & 18.7 & 4.3 \\
\hline $\begin{array}{l}\text { Individual - } \\
\text { 2nd time } \\
\text { point }\end{array}$ & total_MLS_ADD & 1.2 & 0.1 & 0.0 & 3.1 & 1.5 \\
\hline $\begin{array}{l}\text { Individual - } \\
\text { 2nd time } \\
\text { point }\end{array}$ & feed_MLS_ADD & 0.0 & 0.0 & 0.0 & 0.1 & 0.0 \\
\hline $\begin{array}{l}\text { Individual - } \\
\text { 2nd time } \\
\text { point }\end{array}$ & feed_tetracycline_ADD & 9.6 & 6.9 & 3.9 & 18.7 & 5.4 \\
\hline $\begin{array}{l}\text { Individual - } \\
\text { 2nd time } \\
\text { point }\end{array}$ & parenteral_tetracycline_ADD & 1.6 & 2.0 & 0.0 & 4.0 & 1.4 \\
\hline $\begin{array}{l}\text { Individual - } \\
\text { 2nd time } \\
\text { point }\end{array}$ & parenteral_MLS_ADD & 1.2 & 0.0 & 0.0 & 3.0 & 1.5 \\
\hline $\begin{array}{l}\text { Individual - } \\
\text { 2nd time } \\
\text { point }\end{array}$ & parenteral_phenicol_ADD & 0.2 & 0.0 & 0.0 & 3.0 & 0.7 \\
\hline $\begin{array}{l}\text { Individual - } \\
\text { 2nd time } \\
\text { point }\end{array}$ & parenteral_sulfonamide_ADD & 0.1 & 0.0 & 0.0 & 3.0 & 0.4 \\
\hline $\begin{array}{l}\text { Individual - } \\
\text { 2nd time } \\
\text { point }\end{array}$ & parenteral_betalactams_ADD & 0.0 & 0.0 & 0.0 & 0.0 & 0.0 \\
\hline $\begin{array}{l}\text { Individual - } \\
\text { 2nd time } \\
\text { point }\end{array}$ & parenteral_fluoroquinolones_ADD & 0.0 & 0.0 & 0.0 & 0.0 & 0.0 \\
\hline $\begin{array}{l}\text { Pen - } 1 \mathrm{st} \\
\text { time point }\end{array}$ & Total_ADD & 903.6 & 118.2 & 0.0 & 9469.5 & 2030.6 \\
\hline
\end{tabular}




\begin{tabular}{|c|c|c|c|c|c|c|}
\hline $\begin{array}{l}\text { Sample } \\
\text { type }\end{array}$ & Variable & mean & median & $\min$ & $\max$ & sd \\
\hline $\begin{array}{l}\text { Pen - 1st } \\
\text { time point }\end{array}$ & Total_feed_ADD & 591.2 & 38.1 & 0.0 & 8212.5 & 1710.9 \\
\hline $\begin{array}{l}\text { Pen - } 1 \text { st } \\
\text { time point }\end{array}$ & Total_parenteral_ADD & 312.4 & 21.0 & 0.0 & 1326.0 & 419.9 \\
\hline $\begin{array}{l}\text { Pen - 1st } \\
\text { time point }\end{array}$ & total_tetracycline_ADD & 702.1 & 64.9 & 0.0 & 8230.5 & 1700.4 \\
\hline $\begin{array}{l}\text { Pen - } 1 \text { st } \\
\text { time point }\end{array}$ & total_MLS_ADD & 1.2 & 0.0 & 0.0 & 18.0 & 3.7 \\
\hline $\begin{array}{l}\text { Pen - 1st } \\
\text { time point }\end{array}$ & feed_MLS_ADD & 0.7 & 0.0 & 0.0 & 12.0 & 2.4 \\
\hline $\begin{array}{l}\text { Pen - 1st } \\
\text { time point }\end{array}$ & feed_tetracycline_ADD & 590.5 & 38.1 & 0.0 & 8212.5 & 1711.1 \\
\hline $\begin{array}{l}\text { Pen - 1st } \\
\text { time point }\end{array}$ & parenteral_tetracycline_ADD & 111.7 & 0.0 & 0.0 & 1048.0 & 231.0 \\
\hline $\begin{array}{l}\text { Pen - } 1 \text { st } \\
\text { time point }\end{array}$ & parenteral_MLS_ADD & 0.5 & 0.0 & 0.0 & 12.0 & 2.1 \\
\hline $\begin{array}{l}\text { Pen - } 1 \text { st } \\
\text { time point }\end{array}$ & parenteral_phenicol_ADD & 0.4 & 0.0 & 0.0 & 16.0 & 2.5 \\
\hline $\begin{array}{l}\text { Pen - } 1 \mathrm{st} \\
\text { time point }\end{array}$ & parenteral_sulfonamide_ADD & 1.4 & 0.0 & 0.0 & 27.0 & 5.5 \\
\hline $\begin{array}{l}\text { Pen - 1st } \\
\text { time point }\end{array}$ & parenteral_betalactams_ADD & 0.9 & 0.0 & 0.0 & 24.0 & 4.3 \\
\hline $\begin{array}{l}\text { Pen - 1st } \\
\text { time point }\end{array}$ & parenteral_fluoroquinolones_ADD & 197.5 & 0.0 & 0.0 & 1218.0 & 396.5 \\
\hline $\begin{array}{l}\text { Pen - 2nd } \\
\text { time point }\end{array}$ & Total_ADD & 2113.8 & 1054.6 & 9.9 & 10113.3 & 2959.3 \\
\hline $\begin{array}{l}\text { Pen - 2nd } \\
\text { time point }\end{array}$ & Total_feed_ADD & 1745.5 & 763.3 & 9.9 & 8822.3 & 2560.5 \\
\hline $\begin{array}{l}\text { Pen - 2nd } \\
\text { time point }\end{array}$ & Total_parenteral_ADD & 368.3 & 143.0 & 0.0 & 1367.0 & 451.7 \\
\hline $\begin{array}{l}\text { Pen - 2nd } \\
\text { time point }\end{array}$ & total_tetracycline_ADD & 1880.6 & 1012.5 & 9.9 & 8858.3 & 2546.9 \\
\hline $\begin{array}{l}\text { Pen - 2nd } \\
\text { time point }\end{array}$ & total_MLS_ADD & 8.4 & 6.3 & 0.0 & 33.8 & 8.8 \\
\hline $\begin{array}{l}\text { Pen - 2nd } \\
\text { time point }\end{array}$ & feed_MLS_ADD & 5.7 & 0.0 & 0.0 & 33.4 & 7.6 \\
\hline
\end{tabular}




\begin{tabular}{|c|c|c|c|c|c|c|}
\hline $\begin{array}{l}\text { Sample } \\
\text { type }\end{array}$ & Variable & mean & median & $\min$ & $\max$ & sd \\
\hline $\begin{array}{l}\text { Pen - 2nd } \\
\text { time point }\end{array}$ & feed_tetracycline_ADD & 1739.8 & 751.9 & 9.9 & 8822.3 & 2562.5 \\
\hline $\begin{array}{l}\text { Pen - 2nd } \\
\text { time point }\end{array}$ & parenteral_tetracycline_ADD & 140.9 & 18.0 & 0.0 & 902.0 & 231.4 \\
\hline $\begin{array}{l}\text { Pen - 2nd } \\
\text { time point }\end{array}$ & parenteral_MLS_ADD & 2.7 & 0.0 & 0.0 & 12.0 & 4.1 \\
\hline $\begin{array}{l}\text { Pen - 2nd } \\
\text { time point }\end{array}$ & parenteral_phenicol_ADD & 2.8 & 0.0 & 0.0 & 27.0 & 6.4 \\
\hline $\begin{array}{l}\text { Pen - 2nd } \\
\text { time point }\end{array}$ & parenteral_sulfonamide_ADD & 5.0 & 3.0 & 0.0 & 30.0 & 7.6 \\
\hline $\begin{array}{l}\text { Pen - 2nd } \\
\text { time point }\end{array}$ & parenteral_betalactams_ADD & 7.3 & 3.0 & 0.0 & 46.0 & 10.3 \\
\hline $\begin{array}{l}\text { Pen - 2nd } \\
\text { time point }\end{array}$ & parenteral_fluoroquinolones_ADD & 209.6 & 9.0 & 0.0 & 1251.0 & 418.5 \\
\hline $\begin{array}{l}\text { Pen - 3rd } \\
\text { time point }\end{array}$ & Total_ADD & 1340.0 & 1349.6 & 349.5 & 2520.4 & 741.7 \\
\hline $\begin{array}{l}\text { Pen - 3rd } \\
\text { time point }\end{array}$ & Total_feed_ADD & 1053.0 & 945.1 & 349.5 & 1850.4 & 507.6 \\
\hline $\begin{array}{l}\text { Pen - 3rd } \\
\text { time point }\end{array}$ & Total_parenteral_ADD & 287.0 & 380.8 & 0.0 & 670.0 & 260.0 \\
\hline $\begin{array}{l}\text { Pen - 3rd } \\
\text { time point }\end{array}$ & total_tetracycline_ADD & 1228.9 & 1245.2 & 339.7 & 2348.5 & 631.5 \\
\hline $\begin{array}{l}\text { Pen - 3rd } \\
\text { time point }\end{array}$ & total_MLS_ADD & 27.7 & 20.8 & 0.0 & 112.6 & 32.4 \\
\hline $\begin{array}{l}\text { Pen - 3rd } \\
\text { time point }\end{array}$ & feed_MLS_ADD & 25.6 & 15.9 & 0.0 & 112.6 & 32.7 \\
\hline $\begin{array}{l}\text { Pen - 3rd } \\
\text { time point }\end{array}$ & feed_tetracycline_ADD & 1027.5 & 935.1 & 339.7 & 1833.5 & 508.4 \\
\hline $\begin{array}{l}\text { Pen - 3rd } \\
\text { time point }\end{array}$ & parenteral_tetracycline_ADD & 201.4 & 203.0 & 0.0 & 515.0 & 207.3 \\
\hline $\begin{array}{l}\text { Pen - 3rd } \\
\text { time point }\end{array}$ & parenteral_MLS_ADD & 2.1 & 0.0 & 0.0 & 9.0 & 3.5 \\
\hline $\begin{array}{l}\text { Pen - 3rd } \\
\text { time point }\end{array}$ & parenteral_phenicol_ADD & 2.1 & 0.0 & 0.0 & 18.0 & 5.7 \\
\hline $\begin{array}{l}\text { Pen - 3rd } \\
\text { time point }\end{array}$ & parenteral_sulfonamide_ADD & 3.1 & 1.5 & 0.0 & 14.0 & 4.5 \\
\hline
\end{tabular}




\begin{tabular}{|lllllll|}
\hline $\begin{array}{l}\text { Sample } \\
\text { type }\end{array}$ & Variable & mean & median & min & max & sd \\
\hline $\begin{array}{l}\text { Pen - 3rd } \\
\text { time point }\end{array}$ & parenteral_betalactams_ADD & 6.5 & 4.0 & 0.0 & 28.0 & 8.7 \\
\hline $\begin{array}{l}\text { Pen-3rd } \\
\text { time point }\end{array}$ & parenteral_fluoroquinolones_ADD & 71.8 & 6.3 & 0.0 & 582.0 & 180.3 \\
\hline
\end{tabular}

\section{Potential associations between resistome composition and AMD exposures}

Redundancy analysis included investigation of an explanatory variable regarding feedlot, 2 variables regarding timing of sampling, and 14 variables characterizing various aspects of AMD exposures prior to sampling. When including data from all time points for individual animal samples in one model, sampling time point was the only significant variable $(P<0.05)$, but it was only associated with explaining $2.4 \%$ of the constrained variance. For the model investigating data from all time points for pen-floor composite samples, sampling time along with 3 variables describing parenteral exposure to phenicols, macrolides, and sulfonamides were included in the model resulting from step-wise model selection. In all, the sampling time, ADDs for tetracycline exposure, and total ADD exposure were included in the model and were statistically significant $(P<0.05)$, but only accounted for $0.6 \%, 0.2 \%$, and $0.1 \%$ of the constrained variance, respectively. In both of these models, however, unconstrained variance estimates were much greater than constrained variance estimates, suggesting that these results should be interpreted with caution as only a small amount of the variation in the response (resistome) matrix was represented in the model (Legendre and Gallagher, 2001).

Because of the significant shift observed in resistome composition over time, samples collected at the second time point were analyzed separately with RDA. For samples collected from individual animals, the days-on-feed (DOF) variable was the only statistically significant $(P<0.05)$ variable included in the final model, describing $0.2 \%$ of the constrained variance. For pen-floor composite samples, the final RDA model included only 2 statistically significant variables, parenteral MLS ADD and feedlot ID, explaining only $0.4 \%$ and $0.2 \%$ of the constrained variance, respectively. Again, the unconstrained variance for this model was much greater than the constrained variance.

\section{Highly important AMR genes}

Of the 17 genes identified a priori as being important to medicine when expressed in human pathogens, 11 were identified in at least one sample (Supplemental File S7); bla(IMI), bla(KPC), bla(SHV), bla(CPH), bla(NDM), and $m c r$ genes were not identified in any samples. Alignments to these medically important genes accounted for $0.4 \%$ (415K / 93.7M) of all determinants of AMR across all samples. Determinants for betalactamases were the most abundant type of medically important AMR determinant, representing $47 \%$ (195K / 415K) of alignments to these genes. Among these, bla(CTX), bla(OXA) and bla(TEM) were the most abundant, representing 30\% (126K / 415K), 9\% (37K / 415K), and 7\% (31K / 415K) of alignments to medically important AMR determinants, respectively. The alignments to bla(CTX) and 
bla(OXA) genes were fairly evenly distributed across most pen floor composite samples (98/98 and $78 / 98$, respectively), but were more clustered in individual animal samples. This clustering of alignments was even stronger for bla(TEM) among a smaller number samples (7/94 and 13/98 for individual and pen-floor samples, respectively). Interestingly, $90 \%$ of bla(OXA) alignments (33K / 37K) were to OXA-347 (MEGARes gene accession MEG_4750, https://megares.meglab.org). There was also an interesting general trend wherein larger numbers of determinants for these 3 gene groups did not cluster in the same samples. That is, samples that had larger number of alignments for one these genes [bla(CTX), bla(OXA), or bla(TEM)] did not have larger numbers of alignments for the other two. Enzymes encoded by these gene determinants are important in members of the ESBL group. bla(OXA) genes have become medically important because they encode for Class $D$ betalactamase enzymes that are active against cephalosporins and carbapenems (Tooke et al., 2019). While these have been commonly identified in Acinetobacter species, bla(OXA) genes can be found in a variety of bacteria. All alignments to the bla(CTX) group were to one of three MEGAREs gene accessions (MEG_2378, MEG_2430, or MEG_2435), which are variants of the CTX-M-9 subgroup. These ESBL belong to Ambler class A beta-lactamases which have become a medical concern in Enterobacterciae isolates (Bonnet, 2004).

The $v g b \mathrm{~A}$ (streptogramin B esterase), vat (streptogramin A 0-acetyltransferase), and $v g a$ (multidrug ABC efflux pump) genes confer resistance to quinupristin-dalfopristin (Soltani et al., 2000; Jung et al., 2010). This streptogramin class drug combination is especially important for treatment of infections with resistant Gram-positive bacteria, such as methicillin-resistant Staphylococcus aureus (MRSA) and vancomycin-resistant Enterococcus spp (VRE). Alignments to this group of genes were the second most abundant among those identified among the set of medically important AMR genes investigated a priori. Collectively, they represented $37 \%$ (153K / $415 \mathrm{~K}$ ) of alignments to the subset of medically important genes and were identified in $82 \%(158 / 192)$ of all samples. Alignments to $\mathrm{vgbA}$ were the most common among the streptogramin class AMR genes, and the second most abundant among the subset of medically important AMR genes ( $22 \%$ of medically important AMR genes, $92 \mathrm{~K} / 415 \mathrm{~K}$ ). The identification of $v g b A$, vat and $v g a$ were co-located in $24 \%$ (23/94) individual animal samples, and 59\% (58/98) of penfloor composite samples.

Other medically important genes were more sparsely identified in the sample set [cfr, bla(SME), bla(CMY), bla(IMP), and bla(GES)]. Reads aligning to $c f r$ were distributed among the sample set, especially those for $c f r A$, whereas reads aligning to the other ESBL genes listed [bla(SME), bla(CMY), bla(IMP), and bla(GES)] were clustered within a few samples (Supplemental File S7).

\section{Microbiome composition}

Across samples from individual animals, taxa from 29 phyla, 63 classes, and 100 orders were represented (Supplemental File S8). Three phyla (Proteobacteria, Firmicutes, and Bacteroidetes) accounted for over $93 \%$ of all normalized counts $(45.4 \%, 36.9 \%$, and $10.9 \%$, respectively), and each of these phyla were primarily comprised of a single, dominant taxonomic class (Figure 3). Members of the class Gammaproteobacteria comprised over $99 \%$ of all Proteobacteria, while Clostridia made up $75 \%$ of all Firmicutes, and Bacteroidia represented $89.8 \%$ of all Bacteroidetes. At the level of order, 
Pseudomonadales (44.7\%), Clostridiales (26.9\%), Bacteroidales (8.7\%), Lactobacillales (6.6\%), RF39 (2.7\%) and Flavobacteriales (2.0\%), and Enterobacteriales (1.1\%) were the most abundant and combined to represent nearly $93 \%$ of the microbial community. The remaining 93 orders each made up less than $1 \%$ of the overall community.

In pen-floor composite samples, taxa from 25 phyla, 73 classes, and 113 orders were represented. Like individual animal samples, Firmicutes (46.5\%), Bacteroidetes (21.1\%), and Proteobacteria $(20.1 \%)$ were the three most abundant phyla, albeit in a different order of relative abundance. Additionally, Actinobacteria and Tenericutes were more abundant within pen-floor composite samples and accounted for $5.0 \%$ and $4.9 \%$ of the microbial community, respectively (Figure 3 ). As was the case for individual animal samples, the three most abundant phyla were predominantly comprised of a single class. Clostridia comprised $74.7 \%$ of all Firmicutes, while Gammaproteobacteria made up $99.4 \%$ of all Proteobacteria, and Bacteroidia represented $80.7 \%$ of all Bacteroidetes. At the order level, Pseudomonadales (45.3\%), Clostridiales (26.7\%), Bacteroidales (8.6\%), Lactobacillales (6.5\%), RF39 (2.6\%), Flavobacteriales (2\%), and Enterobacteriales (1.1\%) were the most abundant and comprised over $93 \%$ of the microbial community (Figure 4). The remaining 140 orders each represented less than $1 \%$ of the overall community.

\section{Changes in microbiome composition over time}

There were no significant changes in richness or Shannon's diversity indices over time within microbial communities from individual animal samples, but there was a significant decrease in the richness of microbial classes and orders in pen-floor composite samples over time $(P<0.05)$. At arrival, pen-floor composite microbial communities contained an average of 20.2 classes and 27.3 orders, which decreased over time to 18.6 and 25.3, respectively (Supplemental File S8). As demonstrated in the resistome, ANOSIM confirmed that microbial community composition shifted significantly between the first and second time point. Likewise, individual animals had greater shifts in community composition (phylum: ANOSIM R = 0.19, $P<0.01$; class: ANOSIM R=0.21, $P<0.01$; order: ANOSIM R=0.22, $P<0.01$ ) then pen level communities (phylum: ANOSIM R $=0.08, P=0.01$; class: ANOSIM R=0.12, $P<0.01$; order: ANOSIM R= 0.14, $\mathrm{P}<0.01$; Figure 4).

Of the 10 phyla in individual animal samples with an average relative abundance over $1 \%$, seven had significant changes in their relative abundance between sampling time points ( $P$-value $<0.05)$. Bacteroidetes, Proteobacteria, and Spirochaetes significantly increased in relative abundance between first and second time point, while Firmicutes, Cyanobacteria, Actinobacteria, and Verrucomicrobia all decreased $(P<0.05)$ (Supplemental File S9). Of the 11 phyla in pen-floor composites with an average relative abundance over $1 \%$, only six significantly changed in relative abundance from the first to second time point. Tenericutes, Spirochaetes, and Euryarchaeota significantly increased in abundance over time, while Cyanobacteria, Actinobacteria, and Verrucomicrobia all decreased $(P<0.05)$ (Supplemental File S10). 


\section{Potential associations between microbiome composition and AMD exposures}

Parallel to the RDA of variance of the resistome composition, RDA of the microbiome composition investigated the effects of 17 explanatory variables, including 14 variables characterizing AMD exposures. Analysis of individual animal samples from both time points identified sampling time, feedlot ID, and in-feed MLS ADD as statistically significant $(P<0.05)$, but only explained $1.2 \%, 0.6 \%$, and $0.2 \%$ of the constrained variance at the phylum level, respectively. For the RDA of pen-floor composite samples from all 3 time points, DOF, total parenteral ADD exposures, and parenteral sulfonamide ADD were statistically significant $(P<0.05)$, but only explained $1 \%, 0.3 \%$, and $0.3 \%$ of the constrained variance, respectively.

When analyzing the samples collected at the second time point separately, $1.3 \%$ of the constrained variance of the microbiome of individual animal samples was statistically significantly explained by feedlot ID $(P<0.05)$. For pen-floor composite samples, the variables feedlot ID and total ADD exposure were statistically significant $(P<0.05)$ in the RDA of samples collected at the second time point, explaining $1.2 \%$ and $0.5 \%$ of the constrained variance at the phylum level, respectively.

\section{Discussion}

This unique study used data from state-of-the art target-enriched metagenomic sequencing and $16 \mathrm{~S}$ rRNA gene sequencing to investigate critically important questions about factors affecting the promotion of AMR in beef cattle. Results suggested that AMD exposures in beef feedlot cattle do not strongly affect the fecal resistome or microbiome. Despite examination of 14 permutations of AMD exposure in this population that were derived from exceptionally detailed records of AMD exposures in individuals and in groups, these exposure variables were only significantly associated with explaining $<1 \%$ of the variance in the composition of the resistome or microbiome. The factor most strongly associated with resistome and microbiome composition was time in the feedlot, the majority of variability in the resistome composition remained unexplained. Indeed, the variance in the microbiome and resistome that can be explained by the exposure data in this study, or constrained variance, was much smaller than the variance that could not be modeled, unconstrained variance, so results should be interpreted with caution. This study was one of the largest conducted to date regarding the potential anthropogenic impact of AMD exposures typical of those used throughout the beef industry in North America as a promoter of AMR, as assessed using genomic sequencing. These findings add to the growing evidence suggesting that total AMD exposure is not associated with large shifts in the resistome or microbiome of cattle (Vikram et al., 2017; Doster et al., 2018; Rovira et al., 2019).

The higher prevalence of tetracycline, MLS, and aminoglycoside AMR determinants within the cattle resistome, and of Bacteroidetes, Firmicutes, and Proteobacteria within the fecal microbiome of cattle is well documented (e.g. Ghosh and LaPara, 2007; Chen et al., 2008; Platt et al., 2008; Kyselková et al., 2015; Noyes et al., 2016b, 2017; Doster et al., 2018; Rovira et al., 2019). As such, their dominance within 
individual feedlot cattle and pen-floor composite samples collected in this study was unsurprising. Similarly, a greater variation in resistome and microbiome composition within individual animals upon feedlot arrival compared to pen-floor composite samples and later timepoints was expected, as cattleassociated microbial communities are influenced by the multitude of environmental pressures involved with transportation to a feedlot, initial processing, and diet changes typical of feedlot arrival (Noffsinger et al., 2015). Similar shifts in the resistome and microbiome diversity and composition of feces over time have been documented in previous studies of beef cattle during their transition to the feedlot environment (Checkley et al., 2008; Beukers et al., 2015; Noyes et al., 2016b; Doster et al., 2018). These changes are also consistent with the broader conclusion that the function and composition of host-associated microbial communities are significantly influenced by environmental factors (Shafquat et al., 2014). In particular, shifts in microbial community structure resulting from diet changes are well-documented (e.g., ljaz et al., 2018, Singh et al. 2017, Youngblut et al., 2019), and it follows that shifts in resistome and microbiome composition observed in our study would occur as cattle and their associated microbial communities adapted to feedlot environmental pressures.

Despite the perception that AMD use in food animals is a contributor to AMR and treatment failure in humans, there is a paucity of data documenting that these AMD exposures in animals significantly change the resistome, and that resistance determinants are systematically transferred to humans through direct and indirect transmission routes (Robinson et al., 2016; Williams-Nguyen et al., 2016). Concerns regarding anthropogenic promoters of AMR have been especially strong regarding genes that can be found in medically important human pathogens, such as those of ESKAPE pathogens (Pendleton et al., 2013; Santajit and Indrawattana, 2016). The use of third generations cephalosporins, fluoroquinolones, and extended spectrum macrolides in cattle have been noted by some critics as being particularly concerning regarding risks to public health, and antimicrobial resistance determinants for these classes of AMDs, including those that are of high concern when found in medically important human pathogens (Supplemental File S7) were identified in these samples. However, antimicrobial use was not associated with the resistome composition or changes over the feeding period. Additionally, resistance determinants were identified for a number of important classes of AMDs that are not approved for use in cattle (e.g., aminoglycosides, carbapenems, streptogramins, lincosamides, linezolid, and pleuromutilins), and therefore antimicrobial use practices cannot directly explain the presence of these important AMR determinants.

We acknowledge that our study faces the same limitations of many high-throughput sequencing studies. Given the understated nature of the effects of AMD exposure, sequencing depth could have been inadequate to fully characterize the subtle dynamics occurring in low abundance features. However, use of target-enriched sequencing greatly enhanced depth of sequencing that can be efficiently achieved by typical shotgun sequencing, and numbers of reads associated with the resistome that were analyzed in this study are multiple logs greater than in previous studies (Noyes et al., 2016b, 2017; Weinroth et al., 2018; Rovira et al., 2019). The impact of this target-enrichment approach can noted by the numbers of sequencing reads aligning to medically important genes in this study, which were much greater in number and allowed identification of broader range of important yet rare features than were identified in previous 
studies investigating the resistome in feedlot cattle (Noyes et al., 2016b; Weinroth et al., 2018; Rovira et al., 2019). Further, while this study serves as an example of how the use of metagenomics can produce significant and complimentary results from archived samples, it should be noted that samples were originally processed for aerobic culture, and stored in Cary Blair media in a refrigerator prior to freezing. Therefore, our internal validity is sound, but comparisons to external studies using different methods of sample preprocessing should be made with caution.

\section{Conclusions}

A thorough search of the current literature yielded no other metagenomic studies investigating the impact of AMD exposure in beef feedlot cattle on the structure and function of microbial communities. This study substantiates evidence that prior AMD exposure may exert a subtle effect on the microbiome and resistome of feedlot cattle within an ecological context. These results further our understanding of how herd management decisions can influence the microbiome and the resistome, and provide data to help identify practices that maintain the critical balance between the benefits of AMD use and the risk of AMR emergence. As we learn to better manage AMR through livestock production practices, metagenomic analysis will be a critical tool for incorporating a holistic perspective into community-wide changes.

\section{List Of Abbreviations}

ADD - Animal defined daily dose

AMD - Antimicrobial drug

AMR - Antimicrobial resistance

ASV - Amplicon sequence variants

CDC - Centers for Disease Control

CSS - Cumulative sum scaling

DOF - Days on feed

MDR - Multi-drug resistance

MFS - Major facilitator superfamily

MLS - Macrolide-lincosamide-streptogramin

NGS - Next-generation sequencing

RDA - Redundancy analysis 
WHO - World Health Organization

\section{Declarations}

\section{Ethics approval and consent to participate}

Feedlots were recruited for participation by authors working for a veterinary consulting company (Feedlot Health Management Services, Okotoks, Alberta, Canada; FHMS), and owners of the four participating feedlots gave explicit permission to work on the premises and to sample cattle that were enrolled in the study. All cattle handling and sampling procedures were approved prior to the initiation of the study by the Animal Care Committee of the University of Calgary (Protocol Number M07031).

\section{Consent for publication}

Not applicable; no data regarding individual people were included in this research.

\section{Availability of data and materials}

All sequence reads were made available through BioProject PRJNA755709 at the NCBI Sequence Read Archive. The code and instructions used in bioinformatic and statistical analyses can be found at this GitHub repository: https://github.com/Microbial-Ecology-Group/Antimicrobial-drug-use-effect-onmicrobiome-and-resistome-of-beef-feedlots.

\section{Competing interests}

The authors declare that they have no competing interests.

\section{Funding}

This research was funded by grants from the USDA National Institute of Food and Agriculture (201568003-23048), Advancing Canadian Agriculture and Agri-Food (ACAAF) Program, Alberta Beef Producers (0007-038RDB); Canadian Cattlemen's Association - Beef Cattle Research Council (BCRC 6.41), and by Texas A\&M University.

\section{Authors' contributions}

$\mathrm{PM}, \mathrm{SG}, \mathrm{TM}, \mathrm{SH}$, and $\mathrm{CB}$ conceived the original research, organized and oversaw all aspects of its conduct, including collection of fecal samples and detailed antimicrobial drug use records from commercial beef feedlots. PM and KB conceived and oversaw all aspects of the follow-up research regarding metagenomic sequencing of fecal samples collected during the original research. SG and NN assisted in analyzing and preparing the antimicrobial drug exposure data for use in this study. JP and CA conducted laboratory procedures to extract DNA and prepare target-enriched shotgun sequencing libraries. ED analyzed the metagenomic sequencing data, conducted statistical analyses, and prepared 
initial drafts of the manuscript. PM, KB, SG, and LP edited and prepared the final manuscript. All authors read and approved the final manuscript.

\section{Acknowledgements}

Not applicable.

\section{References}

1. Benedict, K. M., Gow, S. P., Checkley, S., Booker, C. W., McAllister, T. A., and Morley, P. S. (2013). Methodological comparisons for antimicrobial resistance surveillance in feedlot cattle. $B M C$ Veterinary Research 9, 216. doi:10.1186/1746-6148-9-216.

2. Benedict, K. M., Gow, S. P., McAllister, T. A., Booker, C. W., Hannon, S. J., Checkley, S. L., et al. (2015). Antimicrobial Resistance in Escherichia coli Recovered from Feedlot Cattle and Associations with Antimicrobial Use. PLOS ONE 10, e0143995. doi:10.1371/journal.pone.0143995.

3. Benjamini, Y., and Hochberg, Y. (1995). Controlling the False Discovery Rate: A Practical and Powerful Approach to Multiple Testing. Journal of the Royal Statistical Society. Series B (Methodological) 57, 289-300.

4. Beukers, A. G., Zaheer, R., Cook, S. R., Stanford, K., Chaves, A. V., Ward, M. P., et al. (2015). Effect of infeed administration and withdrawal of tylosin phosphate on antibiotic resistance in enterococci isolated from feedlot steers. Front Microbio/ 6. doi:10.3389/fmicb.2015.00483.

5. Bolyen, E., Rideout, J. R., Dillon, M. R., Bokulich, N. A., Abnet, C. C., Al-Ghalith, G. A., et al. (2019). Reproducible, interactive, scalable and extensible microbiome data science using QIIME 2. Nat Biotechnol 37, 852-857. doi:10.1038/s41587-019-0209-9.

6. Bonnet, R. (2004). Growing group of extended-spectrum beta-lactamases: the CTX-M enzymes. Antimicrob Agents Chemother 48, 1-14. doi:10.1128/AAC.48.1.1-14.2004.

7. Checkley, S. L., Campbell, J. R., Chirino-Trejo, M., Janzen, E. D., and McKinnon, J. J. (2008). Antimicrobial resistance in generic fecal Escherichia coli obtained from beef cattle on arrival at the feedlot and prior to slaughter, and associations with volume of total individual cattle antimicrobial treatments in one western Canadian feedlot. Can J Vet Res 72, 101-108.

8. Chen, J., Fluharty, F. L., St-Pierre, N., Morrison, M., and Yu, Z. (2008). Technical note: Occurrence in fecal microbiota of genes conferring resistance to both macrolide-lincosamide-streptogramin $B$ and tetracyclines concomitant with feeding of beef cattle with tylosin. J. Anim. Sci. 86, 2385-2391. doi:10.2527/jas.2007-0705.

9. Clarke, K. R. (1993). Non-parametric multivariate analyses of changes in community structure. Australian Journal of Ecology 18, 117-143. doi:10.1111/j.1442-9993.1993.tb00438.x.

10. DeSantis, T. Z., Hugenholtz, P., Larsen, N., Rojas, M., Brodie, E. L., Keller, K., et al. (2006). Greengenes, a Chimera-Checked 16S rRNA Gene Database and Workbench Compatible with ARB. Appl. Environ. Microbiol. 72, 5069-5072. doi:10.1128/AEM.03006-05. 
11. Doster, E., Lakin, S. M., Dean, C. J., Wolfe, C., Young, J. G., Boucher, C., et al. (2020). MEGARes 2.0: a database for classification of antimicrobial drug, biocide and metal resistance determinants in metagenomic sequence data. Nucleic Acids Res. 48, D561-D569. doi:10.1093/nar/gkz1010.

12. Doster, E., Rovira, P., Noyes, N. R., Burgess, B. A., Yang, X., Weinroth, M. D., et al. (2018). Investigating Effects of Tulathromycin Metaphylaxis on the Fecal Resistome and Microbiome of Commercial Feedlot Cattle Early in the Feeding Period. Front Microbio/ 9. doi:10.3389/fmicb.2018.01715.

13. Ghosh, S., and LaPara, T. M. (2007). The effects of subtherapeutic antibiotic use in farm animals on the proliferation and persistence of antibiotic resistance among soil bacteria. ISME J 1, 191-203. doi:10.1038/ismej.2007.31.

14. Hall, R. M., and Schwarz, S. (2016). Resistance gene naming and numbering: is it a new gene or not? J. Antimicrob. Chemother. 71, 569-571. doi:10.1093/jac/dkv351.

15. Jung, Y.-H., Shin, E. S., Kim, O., Yoo, J. S., Lee, K. M., Yoo, J. I., et al. (2010). Characterization of two newly identified genes, vgaD and vatH, [corrected] conferring resistance to streptogramin $\mathrm{A}$ in Enterococcus faecium. Antimicrob Agents Chemother 54, 4744-4749. doi:10.1128/AAC.00798-09.

16. Kyselková, M., Jirout, J., Vrchotová, N., Schmitt, H., and Elhottová, D. (2015). Spread of tetracycline resistance genes at a conventional dairy farm. Front Microbiol 6, 536. doi:10.3389/fmicb.2015.00536.

17. Lakin, S. M., Dean, C., Noyes, N. R., Dettenwanger, A., Ross, A. S., Doster, E., et al. (2017). MEGARes: an antimicrobial resistance database for high throughput sequencing. Nucleic Acids Res 45, D574D580. doi:10.1093/nar/gkw1009.

18. Legendre, P., and Gallagher, E. D. (2001). Ecologically meaningful transformations for ordination of species data. Oecologia 129, 271-280. doi:10.1007/s004420100716.

19. Noffsinger, T., Lukasiewicz, K., and Hyder, L. (2015). Feedlot Processing and Arrival Cattle Management. Veterinary Clinics: Food Animal Practice 31, 323-340. doi:10.1016/j.cvfa.2015.06.002.

20. Noyes, N. r., Benedict, K. m., Gow, S. p., Booker, C. w., Hannon, S. j., McAllister, T. a., et al. (2015). Mannheimia haemolytica in Feedlot Cattle: Prevalence of Recovery and Associations with Antimicrobial Use, Resistance, and Health Outcomes. J Vet Intern Med 29, 705-713. doi:10.1111/jvim.12547.

21. Noyes, N. R., Benedict, K. M., Gow, S. P., Waldner, C. L., Reid-Smith, R. J., Booker, C. W., et al. (2016a). Modelling considerations in the analysis of associations between antimicrobial use and resistance in beef feedlot cattle. Epidemiology \& Infection 144, 1313-1329. doi:10.1017/S0950268815002423.

22. Noyes, N. R., Weinroth, M. E., Parker, J. K., Dean, C. J., Lakin, S. M., Raymond, R. A., et al. (2017). Enrichment allows identification of diverse, rare elements in metagenomic resistome-virulome sequencing. Microbiome 5, 142. doi:10.1186/s40168-017-0361-8.

23. Noyes, N. R., Yang, X., Linke, L. M., Magnuson, R. J., Dettenwanger, A., Cook, S., et al. (2016b). Resistome diversity in cattle and the environment decreases during beef production. eLife 5, e13195. doi:10.7554/elife.13195. 
24. Paulson, J. N., Stine, O. C., Bravo, H. C., and Pop, M. (2013). Differential abundance analysis for microbial marker-gene surveys. Nat Meth 10, 1200-1202. doi:10.1038/nmeth.2658.

25. Peabody, M. A., Van Rossum, T., Lo, R., and Brinkman, F. S. L. (2015). Evaluation of shotgun metagenomics sequence classification methods using in silico and in vitro simulated communities. BMC Bioinformatics 16, 363. doi:10.1186/s12859-015-0788-5.

26. Pendleton, J. N., Gorman, S. P., and Gilmore, B. F. (2013). Clinical relevance of the ESKAPE pathogens. Expert Rev Anti Infect Ther 11, 297-308. doi:10.1586/eri.13.12.

27. Platt, T. M., Loneragan, G. H., Scott, H. M., Norby, B., Thomson, D. U., Brown, M. S., et al. (2008). Antimicrobial susceptibility of enteric bacteria recovered from feedlot cattle administered chlortetracycline in feed. American Journal of Veterinary Research 69, 988-996. doi:10.2460/ajvr.69.8.988.

28. Ritchie, M. E., Phipson, B., Wu, D., Hu, Y., Law, C. W., Shi, W., et al. (2015). limma powers differential expression analyses for RNA-sequencing and microarray studies. Nucleic Acids Research 43, e47e47. doi:10.1093/nar/gkv007.

29. Rovira, P., McAllister, T., Lakin, S. M., Cook, S. R., Doster, E., Noyes, N. R., et al. (2019). Characterization of the Microbial Resistome in Conventional and "Raised Without Antibiotics" Beef and Dairy Production Systems. Front Microbio/ 10. doi:10.3389/fmicb.2019.01980.

30. Santajit, S., and Indrawattana, N. (2016). Mechanisms of Antimicrobial Resistance in ESKAPE Pathogens. Biomed Res Int 2016, 2475067. doi:10.1155/2016/2475067.

31. Soltani, M., Beighton, D., Philpott-Howard, J., and Woodford, N. (2000). Mechanisms of Resistance to Quinupristin-Dalfopristin among Isolates of Enterococcus faecium from Animals, Raw Meat, and Hospital Patients in Western Europe. Antimicrob Agents Chemother 44, 433-436.

32. The Centers for Disease Control and Prevention. (2019). Antibiotic Resistance Threats in the United States. Atlanta, GA. Available: https://www.cdc.gov/drugresistance/pdf/threats-report/2019-arthreats-report-508.pdf.

33. The Centers for Disease Control and Prevention. (2020). Antimicrobial Resistance - Food and Food Animals. Available: https://www.cdc.gov/drugresistance/food.html.

34. Tooke, C. L., Hinchliffe, P., Bragginton, E. C., Colenso, C. K., Hirvonen, V. H. A., Takebayashi, Y., et al. (2019). $\beta$-Lactamases and $\beta$-Lactamase Inhibitors in the 21st Century. Journal of Molecular Biology 431, 3472-3500. doi:10.1016/j.jmb.2019.04.002.

35. Vikram, A., Rovira, P., Agga, G. E., Arthur, T. M., Bosilevac, J. M., Wheeler, T. L., et al. (2017). Impact of "Raised without Antibiotics" Beef Cattle Production Practices on Occurrences of Antimicrobial Resistance. Appl. Environ. Microbiol. 83, e01682-17. doi:10.1128/AEM.01682-17.

36. Weinroth, M. D., Scott, H. M., Norby, B., Loneragan, G. H., Noyes, N. R., Rovira, P., et al. (2018). Effects of Ceftiofur and Chlortetracycline on the Resistomes of Feedlot Cattle. Appl. Environ. Microbiol. 84, e00610-18. doi:10.1128/AEM.00610-18.

37. World Health Organization. (2015). Antimicrobial resistance: global report on surveillance. Geneva. Available: https://www.who.int/iris/bitstream/10665/112642/1/9789241564748_eng.pdf. 
38. World Health Organization. (2017). Critically important antimicrobials for human medicine - 5th rev. Geneva. Available: https://apps.who.int/iris/bitstream/handle/10665/255027/9789241512220eng.pdf.

39. World Health Organization. (2019). No Time to Wait: Securing the future from drug-resistant infections. Report to the Secretary-General of the United Nations. Available: https://www.who.int/antimicrobial-resistance/interagency-coordination-group/final-report/en/.

\section{Supplementary Files}

Supplemental Files are not available with this version.

\section{Figures}

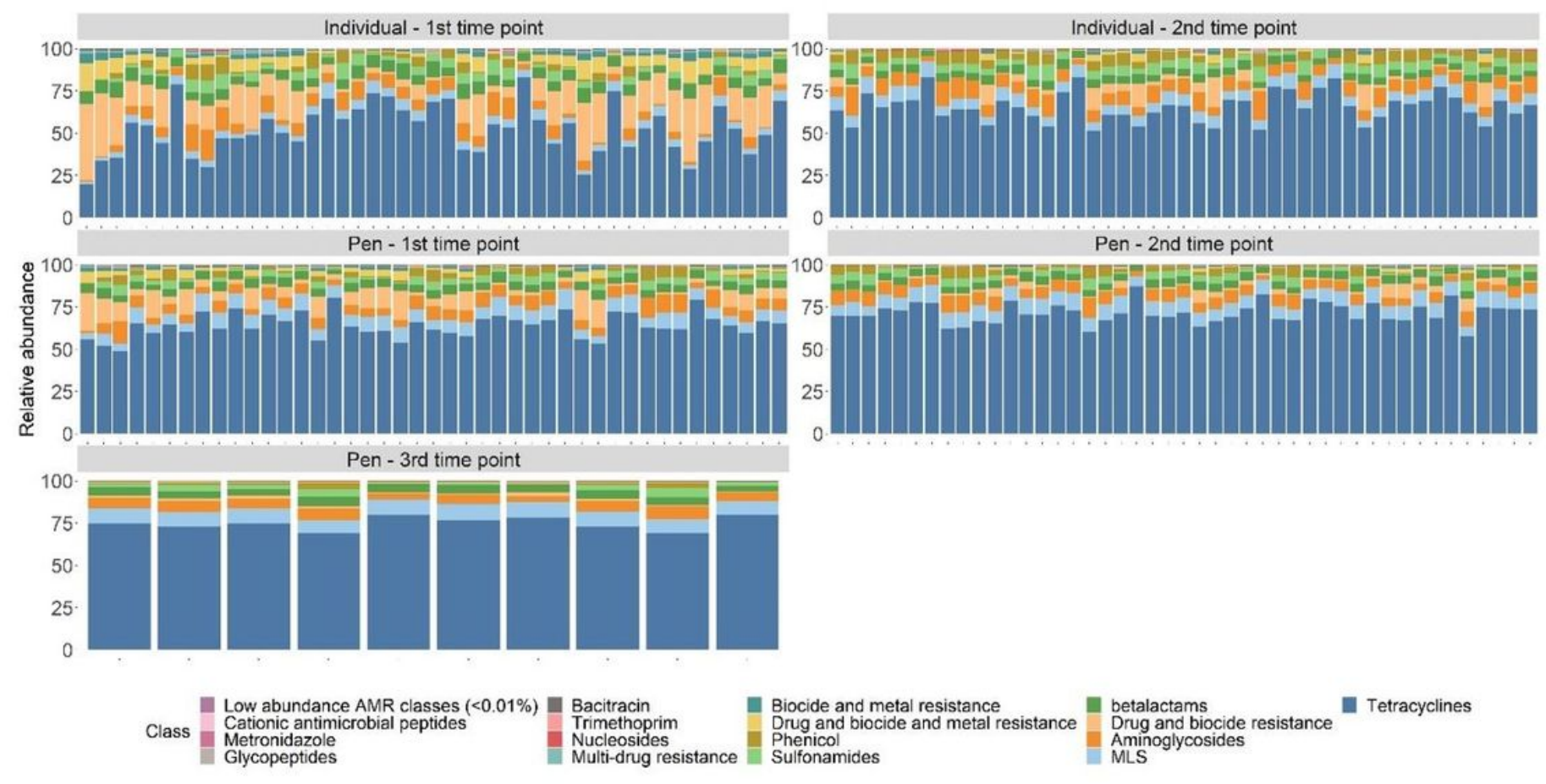

\section{Figure 1}

Resistome composition at the drug class level for all samples by sampling time point. Additionally, a subset of pen-samples were collected at a third time point, just prior to shipment. 
A)
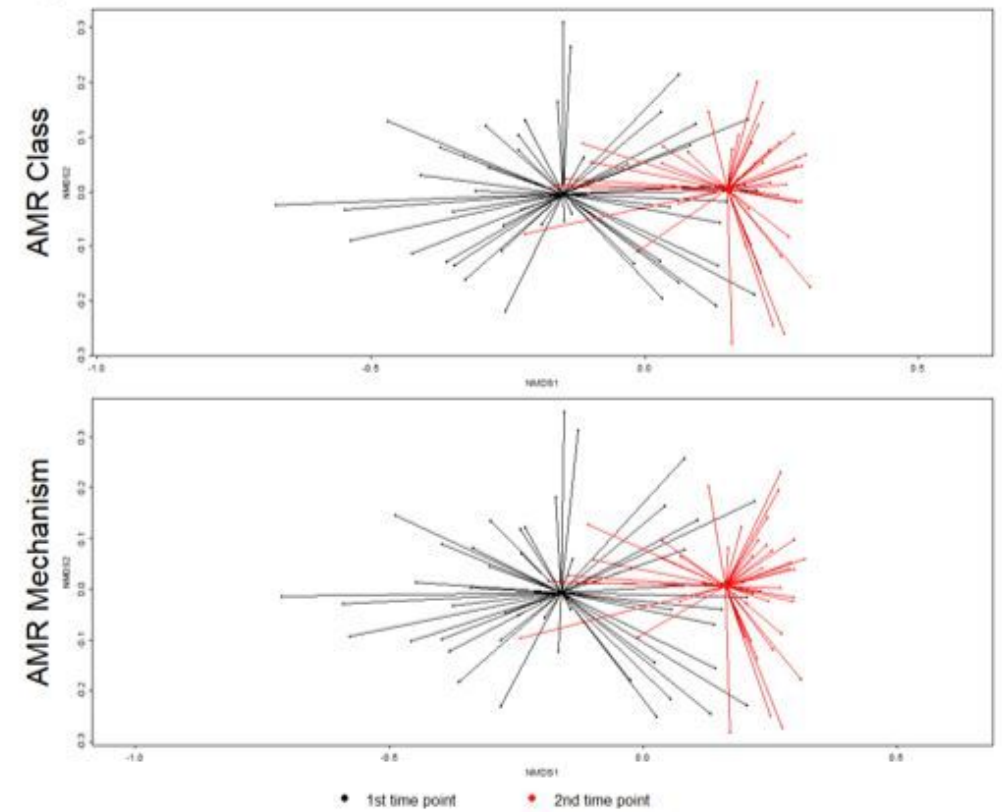

B)
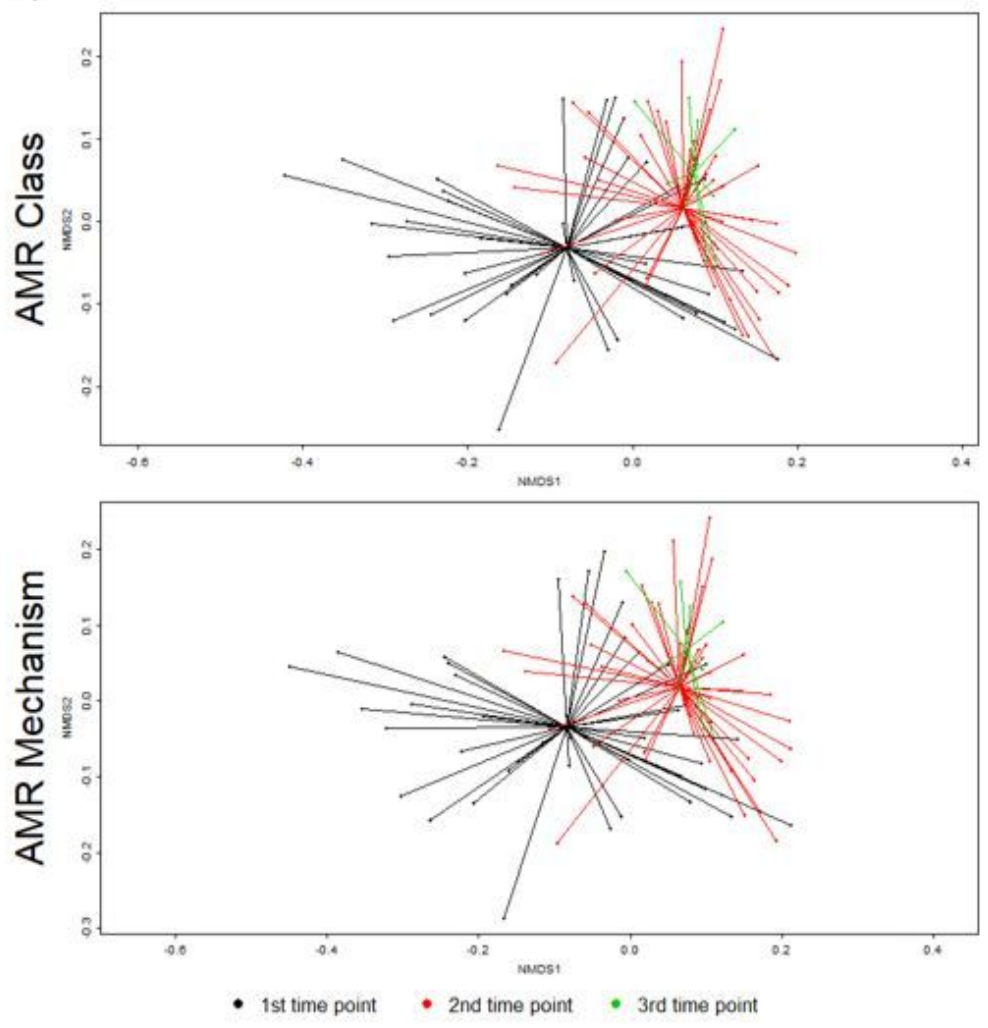

\section{Figure 2}

Ordination comparing resistome composition at the AMR drug class and resistance mechanism, using non-metric multidimensional scaling (NMDS), for the two study groups at arrival and re-handling samples. A) Separation of resistomes in individual animals between sampling time was statistically significant at the class (ANOSIM R $=0.33, P=0.001$ ) and mechanism levels (ANOSIM R $=0.34, P=$ 0.001). B) Separation of resistomes in pen-floor samples between the first and second sampling times 
was statistically significant at the class (ANOSIM R $=0.18, P=0.001$ ) and mechanism levels (ANOSIM R $=0.18, P=0.001$ ).

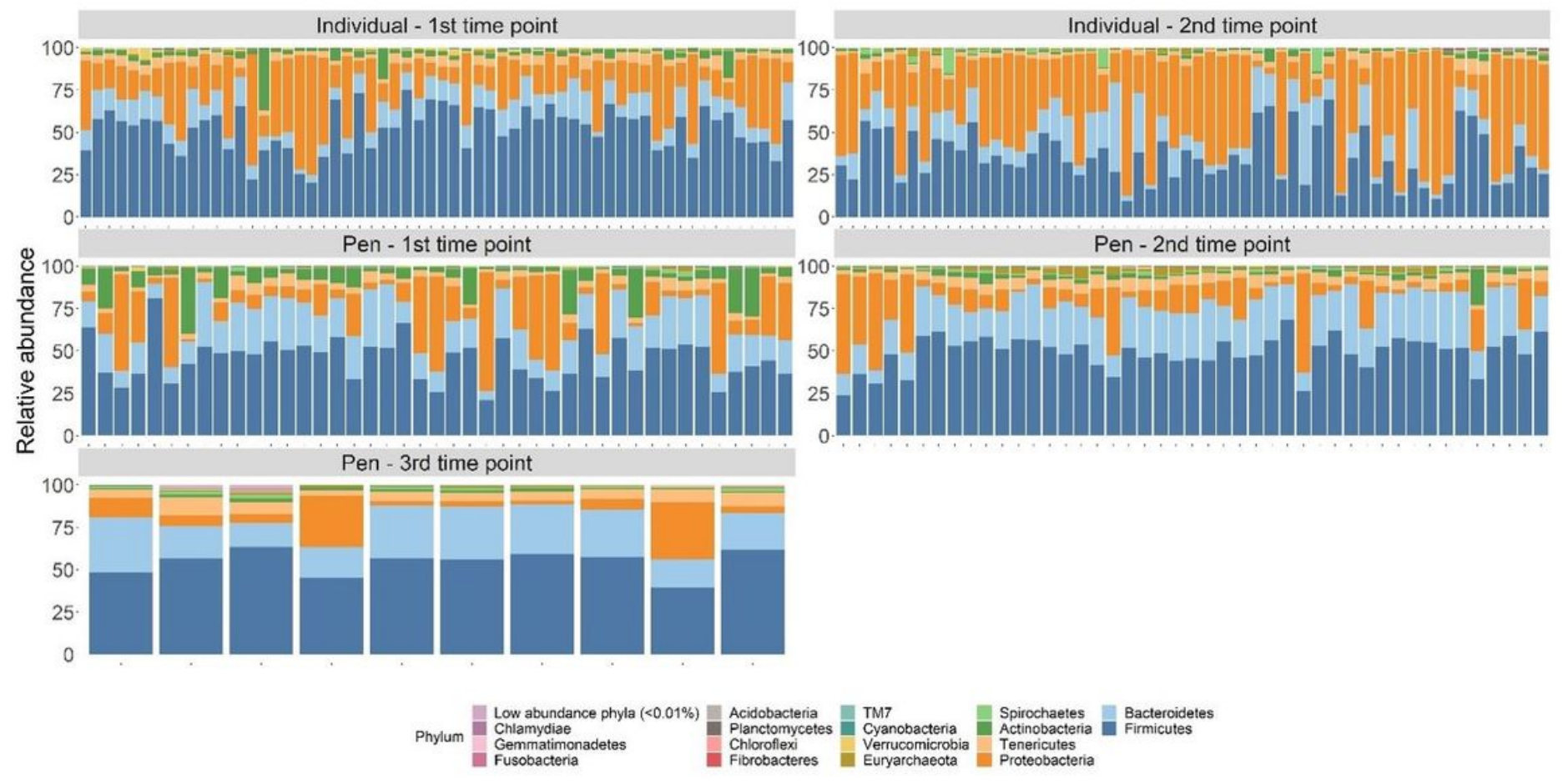

\section{Figure 3}

Microbiome composition at the phylum taxonomic level for all samples by sampling time point; arrival at feedlot, a second time point closer to exit of the feeding period, or just prior to shipment to the abattoir. 

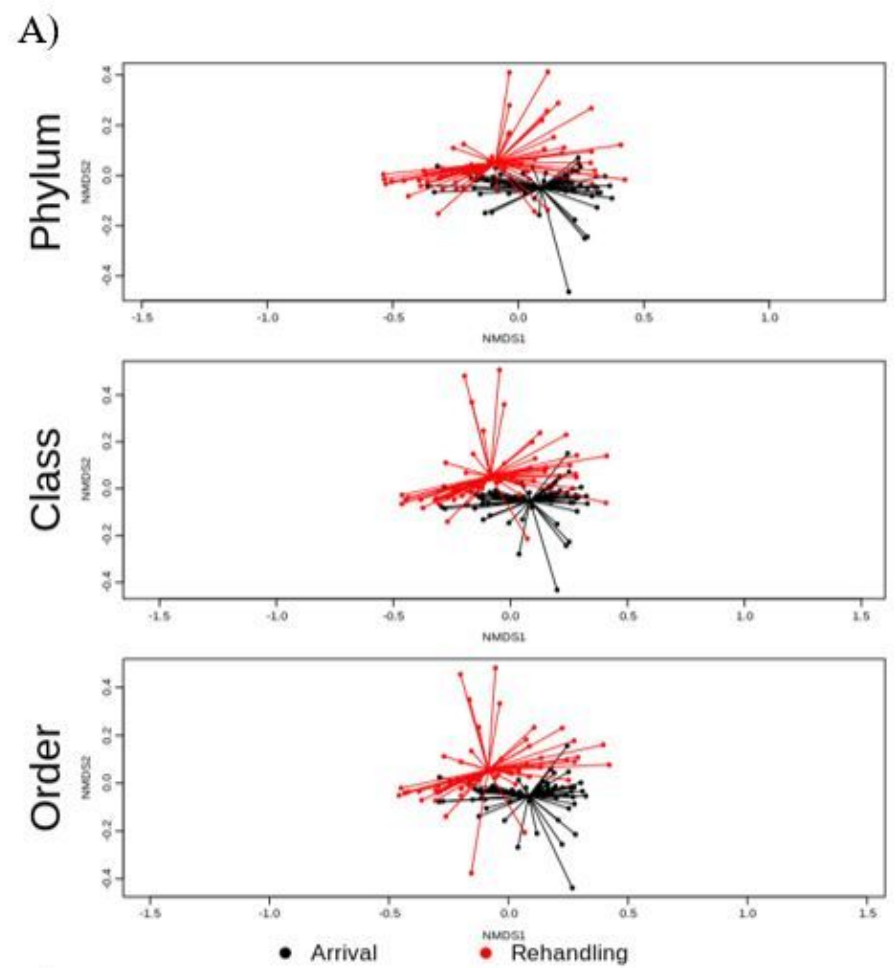

B)
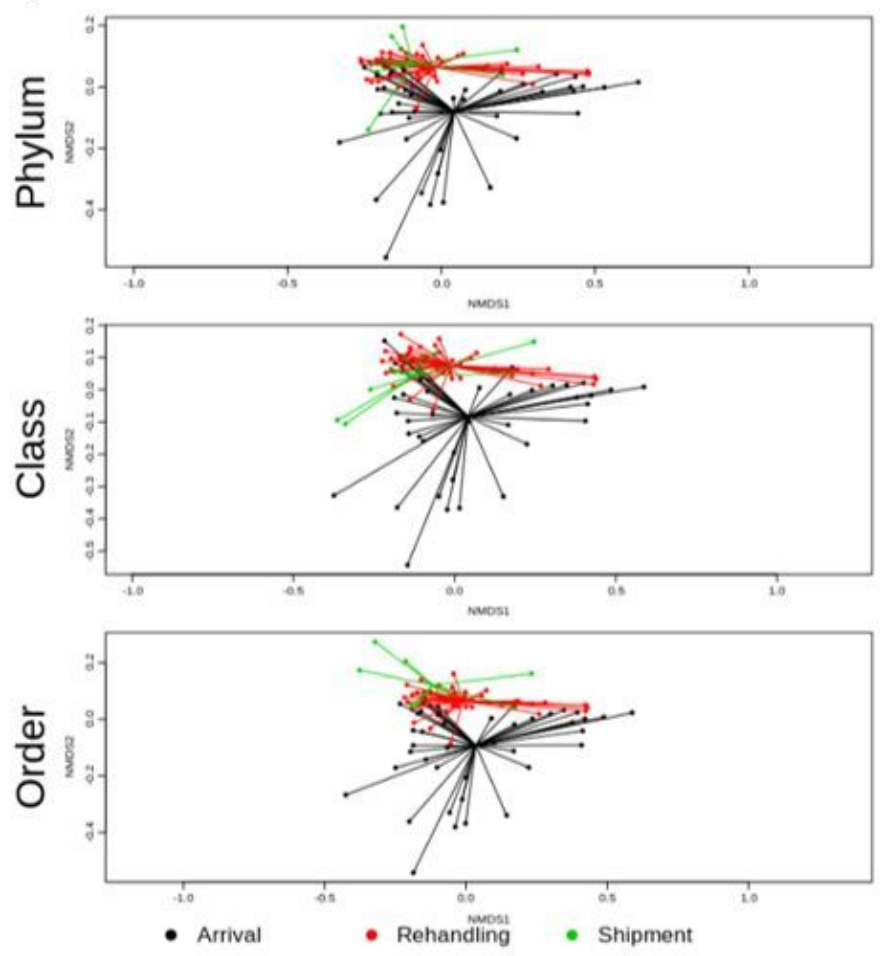

\section{Figure 4}

Ordination comparing microbiome composition at the phylum, class, and order levels using non-metric multidimensional scaling (NMDS), for samples collected at arrival, re-handling, or pen-floor samples collected just prior to shipment. A) In individual animals, the separation of resistomes between sampling time was statistically significant at the phylum (ANOSIM R $=0.19, P<0.01$ ), class (ANOSIM R=0.21, $P<$ 0.01 ), and order (ANOSIM R= 0.22, P < 0.01) taxonomic levels. B) In pen-floor samples, resistome 
composition shifted significantly between the first and second sampling time and was statistically significant at the phylum (ANOSIM R $=0.08, P=0.01$ ), class (ANOSIM R=0.12, $P<0.01$ ), and order (ANOSIM R=0.14, $\mathrm{P}<0.01$ ) taxonomic levels. 